\title{
A long-term optical and X-ray ephemeris of the polar EK Ursae Majoris (Research Note)
}

\author{
K. Beuermann ${ }^{1}$, J. Diese ${ }^{2}$, S. Paik ${ }^{3}$, A. Ploch ${ }^{2}$, J. Zachmann ${ }^{2}$, A. D. Schwope ${ }^{4}$, and F. V. Hessman ${ }^{1}$ \\ 1 Institut für Astrophysik, Göttingen, Germany \\ e-mail: [beuermann; hessman]@astro.physik.uni-goettingen.de \\ 2 Max-Planck-Gymnasium, Göttingen, Germany \\ e-mail: diese@gmx.de, e-mail: icarebooten@web.de, e-mail: jzachmann@t-online.de \\ 3 Felix-Klein-Gymnasium, Göttingen, Germany \\ e-mail: swishfugu00@yahoo.co.kr \\ 4 Astrophysikalisches Institut Potsdam, Potsdam, Germany \\ e-mail: aschwope@aip.de
}

Received 26 June 2009 / Accepted 15 August 2009

\section{ABSTRACT}

\begin{abstract}
Aims. We searched for long-term period changes in the polar EK UMa using new optical data and archival X-ray/EUV data. Methods. An optical ephemeris was derived from data taken remotely with the MONET/N telescope and compared with the X-ray ephemeris based on Einstein, ROSAT, and EUVE data. A three-parameter fit to the combined data sets yields the epoch, the period, and the phase offset between the optical minima and the X-ray absorption dips. An added quadratic term is insignificant and sets a limit to the period change.

Results. The derived linear ephemeris is valid over 30 years and the common optical and $X$-ray period is $P=0.0795440225(24)$ days. There is no evidence of long-term $\mathrm{O}-\mathrm{C}$ variations or a period change over the past 17 years $(\triangle P=-0.14 \pm 0.50 \mathrm{~ms})$. We suggest that the observed period is the orbital period and that the system is tightly synchronized. The limit on $\Delta P$ and the phase constancy of the bright part of the light curve indicate that $\mathrm{O}-\mathrm{C}$ variations of the type seen in the polars DP Leo and HU Aqr or the pre-CV NN Ser do not seem to occur in EK UMa. The X-ray dips lag the optical minima by $9.5^{\circ} \pm 0.7^{\circ}$ in azimuth, providing some insight into the accretion geometry.
\end{abstract}

Key words. novae, cataclysmic variables - stars: individual: EK UMa - X-rays: stars

\section{Introduction}

Long-term studies of cataclysmic variables and related objects have shown that variations in the orbital period occur in some of them, which are not understood or badly so (e.g. Beuermann \& Pakull 1984; Pandel et al. 2002; Schwope et al. 2002; Brinkworth et al. 2006; Schwarz et al. 2009). We set out to search for period changes in other eclipsing and non-eclipsing $\mathrm{CV}$ s using remote observations with the MONET/N telescope as part of a secondary school research project.

The polar EK UMa is the 19 mag optical counterpart of the bright EINSTEIN X-ray source 1E1048.5+5421 discovered by Morris et al. (1987). No optical photometry besides that in the discovery paper is in the literature, but an accurate ephemeris based on ROSAT X-ray data was derived by Schwope et al. (1995). We present new optical photometry and supplement the ROSAT X-ray timings by an analysis of the EUVE deep survey DS/S light curves taken in 1994, 1998, and 1999. The combined data allow us to establish a common long-term ephemeris that extends 30 years back to the Einstein era and accounts for a systematic phase offset between the X-ray absorption dips and the optical cyclotron minima. No period change was found, but we derive a tight limit on $\mathrm{O}-\mathrm{C}$ variations and associated period changes over the past 17 years.

\section{Optical observations}

EK UMa was observed remotely with the MONET/N telescope of the University of Göttingen at the McDonald Observatory in eight nights between 26 February 2009 and 20 April 2009. All observations were performed in white light, as was the 1985 discovery light curve of Morris et al. (1987). The optical light curve possesses a bright phase with two maxima that mark the times when one views the cyclotron emission region at large angles to the magnetic field. They are separated by a deep minimum that occurs when one views most directly along the field. Our light curves, of which two examples are displayed in Fig. 1 (bottom panel), are based on relative photometry with respect to our comparison star $\mathrm{C} 1$, which is located $124 \operatorname{arcsec} \mathrm{E}$ and 178 arcsec $\mathrm{N}$ of EK UMa and is about 2 mag brighter ${ }^{1}$. During our runs, EK UMa varied between $\sim 18$ and $\sim 20$ mag, close to what was estimated by Morris et al. (1987). A second comparison star C2, about equal in brightness to the orbital average of EK UMa and $34 \operatorname{arcsec} \mathrm{E}$ and $67 \operatorname{arcsec} \mathrm{N}$, was used to estimate the error in

\footnotetext{
${ }^{1}$ http://cas.sdss.org/dr7/en/tools/ provides the SDSS magnitudes: $\mathrm{EKUMa}=$ SDSS J105135.14+540436.0, $g=18.38, r=$ $18.99, i=19.04, z=19.19 ; \mathrm{C} 1=$ SDSS J105149.27+540733.6, $g=18.58, r=17.11, i=16.30, z=15.86 ; \mathrm{C} 2=\mathrm{SDSS}$ $\mathrm{J} 105138.99+540543.4, g=20.69, r=19.22, i=18.43, z=17.96$.
} 


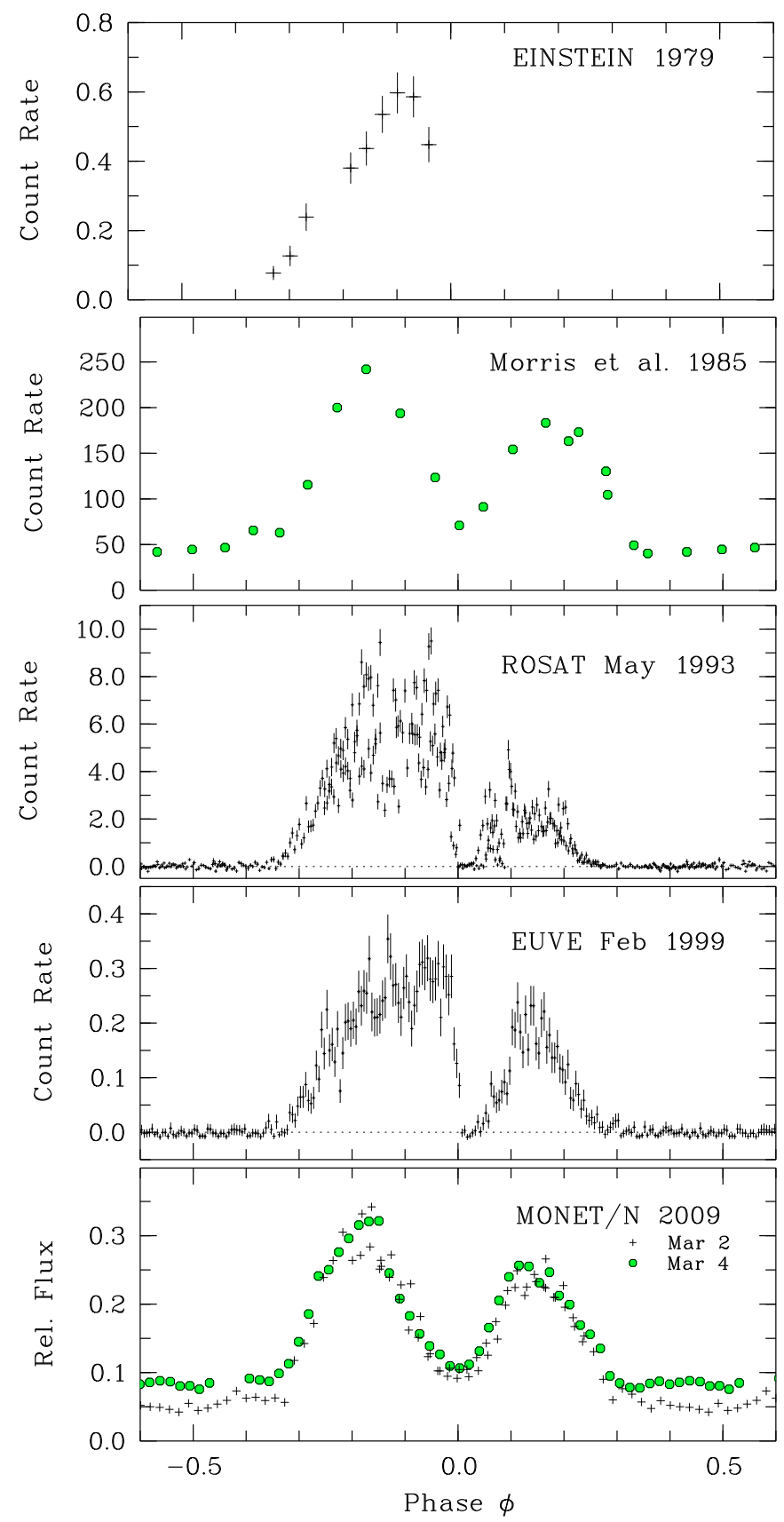

Fig. 1. Phased light curves based on the optical ephemeris of Eq. (1). The Morris et al. (1987) and the MONET/N data were taken in white light. The ordinate for the latter is the flux of EK UMa relative to comparison star $\mathrm{C} 1$, and for all other panels, the ordinate is count rate in units of $\mathrm{s}^{-1}$. The two MONET/N light curves are for cycles $E=0$ and $E=24$.

the photometry of EK UMa. Following these authors, we used the deep central cyclotron minimum as a fiducial mark for the ephemeris.

The time of the minimum $T_{\min }$ and its error were determined by two methods: (1) by a parabola fit to the data points around minimum; and (2) graphically on plots of the individual light curves. Method 1 has the advantage of yielding formal errors for the minimum timings, but suffers from the varying asymmetry of the light curves. In method 2, the center between descending and ascending parts of the light curve is marked at different flux levels and $T_{\text {min }}$ determined by extrapolating any level-dependent shift in the markings to the flux minimum. The timing error is estimated from the scatter of the markings. This method provides insight into the error sources and is the preferred one.

Fifteen MONET/N timings of the central minimum are listed in Table 1, along with the estimated errors. All timings are corrected to the solar system barycenter, corrected for leap seconds, and quoted as BJD(TT). Included in the table is the optical timing of Morris et al. (1987), corrected for leap seconds. Its error of 0.003 days may represent an overestimate as judged from the light curve in Fig. 3 of Morris et al. (1987). Also given in Table 1 are the $\mathrm{O}-\mathrm{C}$ values for the fits discussed below in days and in phase units. Timings with weight $W=0$ are not included in the fits. The band of the observation is quoted as "O" for optical and " $\mathrm{X}$ " for X-ray/EUV data. Note that there is no uncertainty in the cycle numbers given in the first column.

The shapes of our 2009 light curve and the 1985 light curve of Morris et al. are very similar (Fig. 1, bottom panel and second from top). Some night-to-night variability is present and is illustrated by the bright and faint phase intervals varying independently in flux and subtle changes also occurring in the shapes of the light curves (Fig. 1, bottom panel). The bright phase extends over about $65 \%$ of the orbital period, indicating that the main accreting pole is located in the upper hemisphere of the white dwarf. The bright-phase parameters are given in Table 2. The existence of the X-ray absorption dip requires that the inclination exceeds the co-latitude of the accretion spot.

\section{X-ray and EUV light curves}

We obtained soft X-ray light curves from archival data taken with the ROSAT satellite in May 1992, Nov. 1992, and May 1993, and extreme ultraviolet light curves from archival data taken with the EUVE deep survey (DS/S) instrument in Dec. 1994, Dec. 1998, and Jan., Feb., Mar., and Apr. 1999. The Einstein soft X-ray light curve of 1979 was taken from Fig. 2 of Morris et al. (1987). We show the Einstein light curve and phase-folded examples of the ROSAT and EUVE light curves in Fig. 1. The individual light curves of the three ROSAT and the six EUVE observation periods are very similar in shape, with some differences in phase coverage and brightness levels. The May 1993 ROSAT light curve has the best phase coverage of the set of three, and the Feb. 1999 EUVE light curve has the highest count rate of the set of six. The X-ray bright phase intervals coincide approximately with the optical ones and are interrupted by a dip, which occurs when the line of sight crosses the accretion stream at some distance from the white dwarf. We note that the $\mathrm{X}$-ray dips in EK UMa vary in length and possess an ingress that is better defined than the egress. This can be understood in terms of an accretion stream that forms an extended curtain, in which some matter attaches to the field earlier than the bulk of the material and absorbs X-rays after the line of sight has passed the proper accretion stream. We disregard soft X-ray minima with obvious post-dip absorption, a caveat that is particularly important for the EUVE DS/S light curves with their low count rates. The mean timing for each data set (month) of the ROSAT and the EUVE DS/S observations is given in Table 1 along with the estimated timing error. The EINSTEIN data and the Nov. 1992 ROSAT data do not cover the X-ray absorption dip, and the corresponding dip timings are estimated from the well-defined start of the bright phase. The last two and the timing from the ROSAT All-Sky-Survey (RASS) are not included in the fit. 
Table 1. Timings of optical minima and X-ray/EUV dips of EK UMa quoted as terrestrial time at the solar-system barycenter.

\begin{tabular}{|c|c|c|c|c|c|c|c|c|c|c|}
\hline \multirow{2}{*}{$\begin{array}{r}\text { Cycle } \\
\text { number }\end{array}$} & \multirow{2}{*}{$\begin{array}{c}\text { BJD(TT) } \\
\text { (days) }\end{array}$} & \multicolumn{2}{|c|}{ Error } & \multirow[t]{2}{*}{$W$} & \multicolumn{2}{|c|}{$\mathrm{O}-\mathrm{C}$} & \multicolumn{2}{|c|}{$\mathrm{O}-\mathrm{C}-\Delta \mathrm{T}_{\mathrm{o}, \mathrm{x}}$} & \multirow[t]{2}{*}{ B } & \multirow[t]{2}{*}{ Instrument } \\
\hline & & (days) & (phase) & & (days) & (phase) & (days) & (phase) & & \\
\hline-137044 & 43991.898920 & 0.001590 & 0.019989 & 0 & 0.002254 & 0.028331 & 0.000151 & 0.001894 & $\mathrm{X}$ & EINSTEIN $^{(1)}$ \\
\hline-106532 & 46418.943640 & 0.003000 & 0.037715 & 1 & -0.000242 & -0.003038 & -0.000242 & -0.003038 & & Morris et al. \\
\hline-84181 & 48196.835820 & 0.001590 & 0.019989 & 0 & 0.003491 & 0.043886 & 0.001388 & 0.017449 & $\mathrm{X}$ & $R A S S^{(2)}$ \\
\hline-77149 & 48756.187740 & 0.000350 & 0.004400 & 1 & 0.001844 & 0.023188 & -0.000258 & -0.003249 & $\mathrm{X}$ & $R O S A T$ \\
\hline-74652 & 48954.809360 & 0.001590 & 0.019989 & 0 & 0.002040 & 0.025649 & -0.000063 & -0.000788 & $X$ & $\operatorname{ROSAT} T^{(1)}$ \\
\hline-72610 & 49117.238510 & 0.000350 & 0.004400 & 1 & 0.002296 & 0.028867 & 0.000193 & 0.002430 & $\mathrm{X}$ & $R O S A T$ \\
\hline-65258 & 49702.046290 & 0.000500 & 0.006286 & 1 & 0.002423 & 0.030456 & 0.000320 & 0.004019 & $\mathrm{X}$ & $E U V E$ \\
\hline-46777 & 51172.099370 & 0.000550 & 0.006914 & 1 & 0.002422 & 0.030453 & 0.000319 & 0.004016 & $\mathrm{X}$ & $E U V E$ \\
\hline-46359 & 51205.348030 & 0.000580 & 0.007292 & 1 & 0.001681 & 0.021132 & -0.000422 & -0.005305 & $\mathrm{X}$ & $E U V E$ \\
\hline-45992 & 51234.541220 & 0.000350 & 0.004400 & 1 & 0.002215 & 0.027842 & 0.000112 & 0.001405 & $\mathrm{X}$ & $E U V E$ \\
\hline-45673 & 51259.915480 & 0.000350 & 0.004400 & 1 & 0.001931 & 0.024282 & -0.000171 & -0.002155 & $\mathrm{X}$ & $E U V E$ \\
\hline-45247 & 51293.801400 & 0.000460 & 0.005783 & 1 & 0.002098 & 0.026374 & -0.000005 & -0.000063 & $X$ & $E U V E$ \\
\hline-51 & 54888.870790 & 0.000350 & 0.004400 & 1 & -0.000154 & -0.001938 & -0.000154 & -0.001938 & $\mathrm{O}$ & MONET/N \\
\hline-1 & 54892.847380 & 0.000690 & 0.008674 & 1 & -0.000765 & -0.009621 & -0.000765 & -0.009621 & $\mathrm{O}$ & MONET/N \\
\hline 0 & 54892.927690 & 0.000350 & 0.004400 & 1 & 0.000001 & 0.000009 & 0.000001 & 0.000009 & $\mathrm{O}$ & MONET/N \\
\hline 1 & 54893.007530 & 0.000350 & 0.004400 & 1 & 0.000297 & 0.003730 & 0.000297 & 0.003730 & $\mathrm{O}$ & MONET/N \\
\hline 24 & 54894.836640 & 0.000350 & 0.004400 & 1 & -0.000106 & -0.001331 & -0.000106 & -0.001331 & $\mathrm{O}$ & MONET/N \\
\hline 200 & 54908.836120 & 0.000350 & 0.004400 & 1 & -0.000374 & -0.004699 & -0.000374 & -0.004699 & $\mathrm{O}$ & MONET/N \\
\hline 201 & 54908.916110 & 0.000350 & 0.004400 & 1 & 0.000072 & 0.000907 & 0.000072 & 0.000907 & $\mathrm{O}$ & MONET/N \\
\hline 275 & 54914.803220 & 0.000690 & 0.008674 & 1 & 0.000925 & 0.011623 & 0.000925 & 0.011623 & $\mathrm{O}$ & MONET/N \\
\hline 276 & 54914.882060 & 0.000350 & 0.004400 & 1 & 0.000220 & 0.002772 & 0.000220 & 0.002772 & $\mathrm{O}$ & MONET/N \\
\hline 277 & 54914.961360 & 0.000350 & 0.004400 & 1 & -0.000024 & -0.000296 & -0.000024 & -0.000296 & $\mathrm{O}$ & MONET/N \\
\hline 564 & 54937.790620 & 0.000350 & 0.004400 & 1 & 0.000102 & 0.001282 & 0.000102 & 0.001282 & $\mathrm{O}$ & MONET/N \\
\hline 565 & 54937.870370 & 0.000350 & 0.004400 & 1 & 0.000308 & 0.003872 & 0.000308 & 0.003872 & $\mathrm{O}$ & MONET/N \\
\hline 576 & 54938.744750 & 0.000350 & 0.004400 & 1 & -0.000296 & -0.003725 & -0.000296 & -0.003725 & $\mathrm{O}$ & MONET/N \\
\hline 577 & 54938.824110 & 0.000690 & 0.008674 & 1 & -0.000480 & -0.006038 & -0.000480 & -0.006038 & $\mathrm{O}$ & MONET/N \\
\hline 615 & 54941.847300 & 0.000350 & 0.004400 & 1 & 0.000037 & 0.000463 & 0.000037 & 0.000463 & $\mathrm{O}$ & MONET/N \\
\hline
\end{tabular}

(1) Based on the start of the bright phase at $\phi=-0.37 \pm 0.02$ relative to the center of the X-ray dip. Not used in deriving the ephemeris; ${ }^{(2)} R O S A T$ All Sky Survey. Not used in deriving the ephemeris.

\section{Results}

The fifteen new MONET/N timings of 2009 combined with the 1985 timing of Morris et al. (1987) define an alias-free ephemeris with an optical period $P_{\text {opt }}=0.079544025$ (28) days, where the number in brackets refers to the uncertainty in the last digits. This uncertainty arises almost entirely from the large error assigned to the 1985 timing by Morris et al. (1987). Fitting the eight timings from the pointed ROSAT and EUVE data yields $P_{\mathrm{x}}=0.079544022$ (3) days. Both periods agree within the uncertainties with the pre-1995 period of Schwope et al. (1995). There is a significant difference, however, in the epochs of the optical and the X-ray/EUV ephemerides, indicating that the X-ray/EUV dips occur about 3 min later than the optical minima. We proceed with a three-parameter fit to the combined set of 24 optical and X-ray/EUV timings, $T_{\min }=T_{0}+\Delta T_{\mathrm{o}, \mathrm{x}}+P E$. The three parameters are the common period $P$, the epoch $T_{0}$, and an offset $\Delta T_{\mathrm{x}}$, which we subtract from the X-ray/EUV dip times prior to the common fit. Our reference is the optical ephemeris and the corresponding quantity $\Delta T_{\mathrm{o}}$ for the optical timings is set to zero. The resulting linear ephemeris, applicable to the optical and the $\mathrm{X}$-ray data, is

$T_{\min }=2454892.92769(8)+\Delta T_{\mathrm{o}, \mathrm{x}}+0.0795440225(24) E$,

with $T_{\min }$ given as barycentrically corrected terrestrial (ephemeris) times, $\Delta T_{\mathrm{o}} \equiv 0, \Delta T_{\mathrm{x}}=0.00210$ (14) days, and $\chi^{2}$ of 10.2 for 21 degrees of freedom. An added quadratic term $C E^{2}$ in the ephemeris turns out to be insignificant with $C=-(1.1 \pm 3.7) \times 10^{-14}$ days and $\dot{P}=-(2.7 \pm 9.3) \times 10^{-13} \mathrm{~s} \mathrm{~s}^{-1}$.
Table 2. Phases $\phi$ for the the central minima and the lengths of the bright intervals, with $\phi=0$ referring to the optical minimum.

\begin{tabular}{lcccc}
\hline \hline Observation & \multirow{2}{*}{$\phi_{\min / \text { dip }}$} & \multicolumn{3}{c}{ Bright } \\
& & $\phi_{\text {start }}$ & $\phi_{\text {end }}$ & $\phi_{\text {center }}$ \\
\hline Optical 1987 & 0.000 & -0.34 & 0.32 & -0.01 \\
ROSAT 1992/1993 & 0.026 & -0.34 & 0.26 & -0.04 \\
EUVE 1994/1999 & 0.026 & -0.33 & 0.27 & -0.03 \\
MONET/N 2009 & 0.000 & -0.33 & 0.31 & -0.01 \\
\hline
\end{tabular}

(1) Errors typically 0.01, except for the 1987 data, where they are 0.02 .

The error in $C$ limits the period variation over the well-observed time interval from 1992 to 2009 with $\Delta E=77759$ cycles to $\Delta P=2 C \Delta E=-(0.14 \pm 0.50) \mathrm{ms}$.

The offset between the cyclotron minima and the X-ray absorption dips indicates that the magnetic funnel near the white dwarf leads the $\mathrm{X}$-ray absorption region by $9.5^{\circ} \pm 0.7^{\circ}$. The sense of curvature of the stream is as expected if the accreting pole precedes the coupling region in the magnetosphere.

The measured period is the rotation period of the white dwarf, provided the accretion spot does not wander over the white dwarf. The mean start and stop phases of the bright part of the light curve appear constant over 24 years (Fig. 1 and Table 2), and the ensuing lack of a change in the accretion geometry suggests that what we measure is the orbital period and that the system is tightly synchronized. A final decision on the degree of 

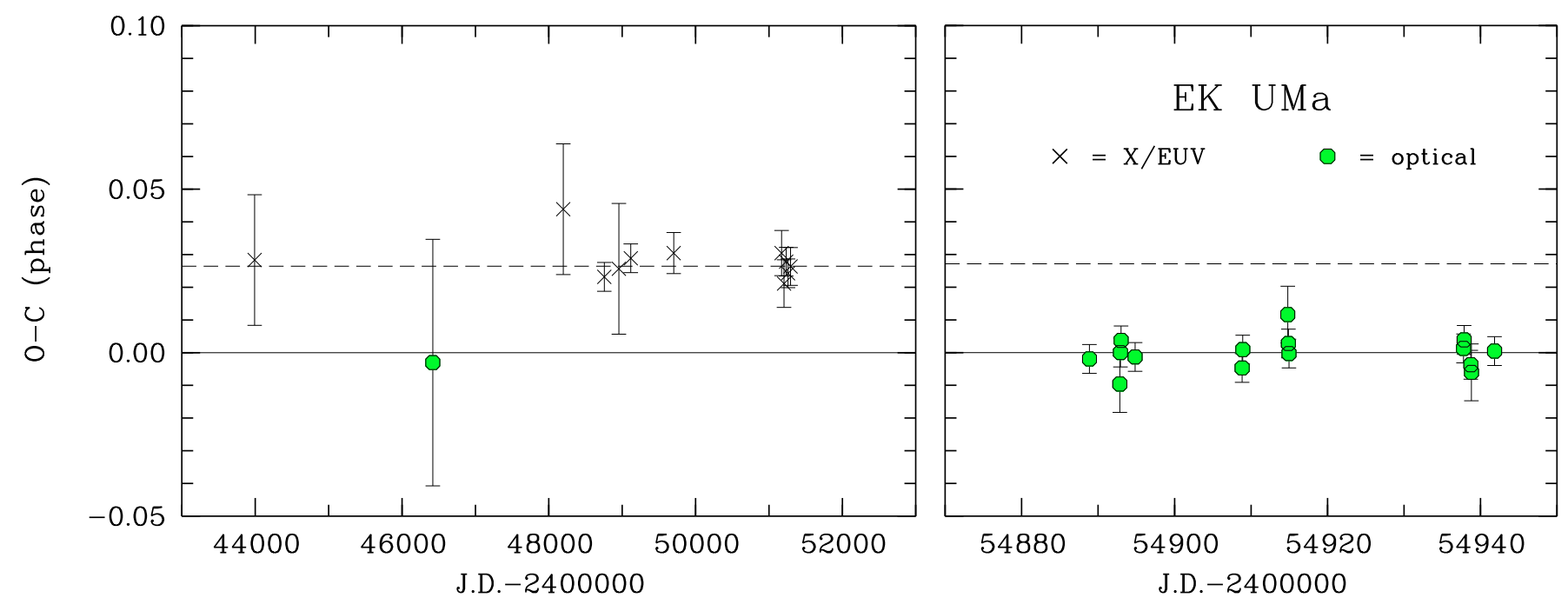

Fig. 2. O - C diagrams for timings of the optical cyclotron minima and the X-ray absorption dips before 2000 (left) and in 2009 (right). The solid and dashed lines represent the linear ephemeris of Eq. (1) with $\Delta T_{\mathrm{o}}=0$ and $\Delta T_{\mathrm{x}} / P=0.0264$, respectively. The scale is changed between the two panels.

synchronization requires long-term spectroscopic observations of emission or absorption lines from the secondary star.

\section{Discussion}

We find that the optical and X-ray data of EK UMa define an ephemeris that is linear over at least 17 years. A quadratic term is insignificant and limits the period variation over this time span to $-1.1<\Delta P<0.9 \mathrm{~ms}$ ( $2-\sigma$ limit). With long-term coverage becoming available for an increasing number of $\mathrm{CVs}$, measuring period variations in the $\mathrm{ms}$ or sub-ms range becomes feasible. In the eclipsing pre-CV NN Ser, Brinkworth et al. (2006) find $\dot{P}=-(9.06 \pm 0.06) \times 10^{-12} \mathrm{~s} \mathrm{~s}^{-1}$ over 45000 cycles and a still higher $\dot{P}=-(2.85 \pm 0.15) \times 10^{-11} \mathrm{~s} \mathrm{~s}^{-1}$ over some 3000 cycles. The eclipsing polars DP Leo (Pandel et al. 2002; Schwope et al. 2002) and HU Aqr (Schwarz et al. 2009) showed decreases in the orbital periods with $\dot{P} \simeq-5 \times 10^{-12} \mathrm{~s} \mathrm{~s}^{-1}$ over 120000 cycles and $\dot{P}=-(7.3 \pm 0.5) \times 10^{-13} \mathrm{~s} \mathrm{~s}^{-1}$ over 60000 cycles, with superposed possibly sinusoidal variations in the latter. A sinusoidal modulation in the $\mathrm{O}-\mathrm{C}$ values has previously been seen in the eclipsing dwarf nova U Gem (e.g. Beuermann \& Pakull 1984). While star spot cycles may account for the quasi-periodic variations, there is no entirely convincing explanation of the secular period changes emphasizing the need for further studies meant to differentiate between typical behavior and idiosyncrasies. We have demonstrated that subtle period variations - or their absence are easily detected using a small, remotely operated telescope.

Acknowledgements. The MONET Monitoring Network of 1.2-m optical telescopes is funded by the Alfried Krupp von Bohlen und Halbach Foundation and provides part of the observation time to astronomical projects at high schools. This research has made use of archival data obtained with the ROSAT X-ray satellite and the EUVE extreme ultraviolet satellite. The data were retrieved from the ROSAT Archive at the MPE at Garching/Munich and the High Energy Astrophysics Science Archive Research Center (HEASARC) at NASA's Goddard Space Flight Center.

\section{References}

Beuermann, K., \& Pakull, M. W. 1984, A\&A, 136, 250

Brinkworth, C. S., Marsh, T. R., Dhillon, V. S., et al. 2006, MNRAS, 365, 287

Morris, S. L., Schmidt, G. D., Liebert, J., et al. 1987, ApJ, 314, 641

Clayton K. L., \& Osborne, J. P. 1994, MNRAS, 268, 229

Cropper, M., Mason, K. O., \& Mukai, K. 1990, MNRAS, 243, 565

Pandel, D., Cordova, F. A., Shirey, R. E., et al. 2002, MNRAS, 332, 116

Schwarz, R., Schwope, A. D., Vogel, J., et al. 2009, A\&A, 496, 833

Schwope, A. D., Beuermann, K., Burwitz, V., Mantel, K.-H., \& Schwarz, R. 1995, Cataclysmic Variables, ASSL, 205, 389

Schwope, A. D., Hambaryan, V., Schwarz, R., Kanbach, G., Gänsicke, B. T. 2002, A\&A, 392, 541 